\title{
Evaluation of Antibody Properties and Clinically Relevant Immunogenicity, Anaphylaxis, and Hypersensitivity Reactions in Two Phase III Trials of Tralokinumab in Severe, Uncontrolled Asthma
}

\author{
Mats Carlsson ${ }^{1}$ - Martin Braddock ${ }^{2} \cdot$ Yuling $\mathrm{Li}^{3} \cdot$ Jihong Wang ${ }^{3} \cdot$ Weichen $\mathrm{Xu}^{3} \cdot$ Nicholas White $^{4} \cdot$ Ayman Megally $^{5}$. \\ Gillian Hunter ${ }^{6}$. Gene Colice ${ }^{5}$
}

Published online: 16 January 2019

(c) The Author(s) 2019

\begin{abstract}
Introduction Tralokinumab is a monoclonal antibody (mAb) that neutralizes interleukin (IL)-13, a cytokine involved in the pathogenesis of asthma.

Objective The objectives of this study were to characterize the potential immunogenic properties of tralokinumab and report data for anti-drug antibodies (ADAs) and hypersensitivity reactions from two phase III clinical trials.

Methods The oligosaccharide structure of tralokinumab, Fab-arm exchange, and ADAs were characterized by standard techniques. Hypersensitivity adverse events (AEs) were evaluated in two pivotal clinical trials of tralokinumab in severe, uncontrolled asthma: STRATOS 1 and 2 (NCT02161757 and NCT02194699).

Results No galactose- $\alpha-1,3$-galactose ( $\alpha$-Gal) epitopes were found in the Fab region of tralokinumab and only $4.5 \%$ of glycoforms contained $\alpha-\mathrm{Gal}$ in the Fc region. Under non-reducing conditions, Fab-arm exchange did not take place with another immunoglobulin ( $\mathrm{Ig}$ ) $\mathrm{G}_{4} \mathrm{mAb}$ (mavrilimumab). However, following glutathione reduction, a hybrid antibody with monovalent bioactivity was detected. ADA incidences (titers) were as follows: STRATOS 1 -every 2 weeks (Q2 W) $0.8 \%$ (26.0), every 4 weeks (Q4 W) 0.5\% (26.0), placebo 0.8\% (52.0); STRATOS 2-Q2 W 1.2\% (39.0), placebo 0.8\% (13.0). Participant-reported hypersensitivity AE rates were as follows: STRATOS 1-Q2 W 25.9\%, Q4 W 25.0\%, placebo 25.5\%; STRATOS 2-Q2 W 13.2\%, placebo 9.0\%. External evaluation for anaphylaxis by Sampson criteria found no tralokinumabrelated severe hypersensitivity or anaphylaxis reactions.

Conclusion Preclinical assessments suggested a low likelihood of immunogenicity for tralokinumab. In STRATOS 1 and 2, ADA incidence was low, no differences were found between tralokinumab-treated and placebo groups in reporting of hypersensitivity reactions, and there were no Sampson criteria-evaluated anaphylaxis events with tralokinumab treatment. Together, the results suggest that tralokinumab treatment would not increase the risk for severe hypersensitivity or anaphylactic reactions.
\end{abstract}

Electronic supplementary material The online version of this article (https://doi.org/10.1007/s40264-018-00788-w) contains supplementary material, which is available to authorized users.

Mats Carlsson

Mats.Carlsson@astrazeneca.com

1 Patient Safety, Global Medicines Development, AstraZeneca, Pepparedsleden 1, Mölndal 431 83, Sweden

2 Global Medicines Development, AstraZeneca, Granta Park, Great Abington, Cambridge CB21 6GH, UK

3 Biopharmaceutical Development, MedImmune, One MedImmune Way, Gaithersburg, MD 20878, USA
4 Clinical Pharmacology, Pharmacometrics and DMPK, MedImmune, Granta Park, Great Abington, Cambridge CB21 6GH, UK

5 Global Medicines Development, AstraZeneca, One MedImmune Way, Gaithersburg, MD 20878, USA

6 Biometrics and Information Sciences, AstraZeneca, Granta Park, Great Abington, Cambridge CB21 6GH, UK 


\section{Key Points}

In vitro characterization of the glycans of tralokinumab and its ability to undergo Fab-arm exchange did not suggest that there would be an increased risk for hypersensitivity and anaphylactic reactions during tralokinumab treatment.

The STRATOS 1 and 2 clinical findings were in line with these in vitro data. Low incidence of ADAs and neutralizing antibodies were reported, and no tralokinumab-related severe hypersensitivity or anaphylaxis reactions were observed according to Sampson criteria.

\section{Introduction}

Tralokinumab is an immunoglobulin (Ig) $\mathrm{G}_{4}$ human monoclonal antibody $(\mathrm{mAb})$ that potently and specifically neutralizes interleukin (IL)-13 [1] by preventing binding to IL-13 receptors (IL-13R) $\alpha 1$ and $\alpha 2$ [2]. IL-13 is a pleiotropic type- 2 cytokine that has a central role in the pathogenesis of some types of asthma [3]. For example, in severe asthma, IL-13 promotes the production of $\mathrm{IgE}$, leading to recruitment of eosinophils and airway remodeling via IL-4R $\alpha$, IL-13R $\alpha 1$, and/or IL-4 $\alpha$ [4]. As part of the ATMOSPHERE late-stage clinical development program [5], tralokinumab was investigated in two pivotal phase III clinical trials, STRATOS 1 and 2 [6,7], for the treatment of severe, uncontrolled asthma. There are safety challenges associated with developing a mAb for clinical use; for example, mAbs may present an immunogenic risk with the potential to induce severe hypersensitivity reactions such as anaphylaxis $[8,9]$. Several factors have been identified that affect the immunogenicity of therapeutic mAbs and potentially increase the risk of hypersensitivity reactions; these include the nature of the mAb [10], the manufacturing process, stability of the $\mathrm{mAb}$, drug excipients, mode of administration, and recipient predisposition [9]. Tralokinumab has two qualities that could hypothetically increase the risk of hypersensitivity reactions and of other adverse events (AEs): the presence of galactose$\alpha-1,3$-galactose ( $\alpha$-Gal) epitopes on the antibody, and the propensity of $\mathrm{IgG}_{4} \mathrm{mAbs}$ to undergo Fab-arm exchange and form bispecific antibodies.

The presence of $\alpha$-Gal epitopes is one of the structural elements of a mAb that may be affected by the manufacturing process, and may influence the potential for developing anaphylactic or hypersensitivity reactions. During production in mammalian cells, mAbs typically undergo glycosylation, a post-transcriptional modification whereby enzymes attach glycans to proteins [10]. The type and the level of glycosylation are associated with the mAb characteristics, the production host cell line, and the cell culture conditions. A functional gene for $\alpha-1,3$-galactosyltransferase, which is the enzyme required for $\alpha$-Gal epitope synthesis, is absent in humans but present in mice [11]. As a result, mAbs that are manufactured using mouse cell lines may be glycosylated with glycans that contain the $\alpha$-Gal epitope. These epitopes are seen as 'foreign' by the human immune system and may elicit a xenoreactive immune response. Such responses have previously been reported with cetuximab, a chimeric mouse-human $\mathrm{IgG}_{1} \mathrm{mAb}$ for the treatment of colorectal cancer, which is produced in the mouse cell line $\mathrm{Sp} 2 / 0$ and contains $\alpha-\mathrm{Gal}$ epitopes [10]. Immune reactions including severe hypersensitivity and anaphylaxis reported in people taking cetuximab are thought to be connected to the $\alpha-\mathrm{Gal}$ epitopes $[10,12]$. Tralokinumab is produced using a mouse myeloma cell line (NSO) that has the ability to attach $\alpha$-Gal to the mAb during glycosylation [13] and could, therefore, contain these epitopes. Consequently, the question arose whether tralokinumab, like cetuximab, might be potentially associated with an increased risk of hypersensitivity or anaphylaxis compared with other mAbs.

Another structural characteristic of a mAb that can affect its immunogenic potential is its $\mathrm{IgG}$ subclass. The $\mathrm{IgG}_{4} \mathrm{mAb}$ has been an isotype of choice for therapeutic mAbs due to limited effector functions [14, 15] and lack of allotypes [16], which both theoretically reduce the potential for hypersensitivity reactions. However, unlike $\operatorname{IgG}_{1}, \mathrm{IgG}_{2}$, and $\mathrm{IgG}_{3}$ isotypes, the core-hinge region sequence of $\mathrm{IgG}_{4}$ allows the inter-heavy chain disulfide bonds in the hinge region to form intra-heavy chain disulfide bonds, resulting in half-antibody molecules $[14,17]$. This half-antibody formation can lead to Fab-arm exchange with other $\mathrm{IgG}_{4}$ antibodies. The resulting $\mathrm{IgG}_{4}$ antibodies are functionally monovalent (i.e., unable to cross-link identical antigens) but structurally bispecific (i.e., able to bind two different antigens) [18]. These functionally bispecific $\mathrm{IgG}_{4}$ antibodies have been proposed to contribute to AEs due to potential undesired specificity $[14,19,20]$. As an example, it has been suggested that Fab-arm exchange of natalizumab (a humanized IgG4 $\kappa$ anti- $\alpha 4$ subunit of integrin $\mathrm{mAb}$ ) may be implicated in the pathogenesis of progressive multifocal leukoencephalopathy (PML), an opportunistic, life-threatening viral disease [19]. Tralokinumab has the typical structure of an $\mathrm{IgG}_{4}$ molecule, with two identical heavy chains and two identical light chains [21], and therefore has the potential to form half-antibodies with endogenous $\operatorname{IgG}_{4}$ antibodies [17].

Therapeutic mAbs can elicit anti-drug antibodies (ADAs) when administered, which are another sign of immunogenicity. The ADAs bind to the mAb, forming immune complexes, which can lead to reduced pharmacological activity, reduced exposure to the mAb, or hypersensitivity reactions. The presence of large, cross-linked 
ADA-mAb immune complexes in the circulation may lead to type III hypersensitivity reactions when these complexes are deposited in tissues such as the kidneys and synovial membranes [22].

The objectives of this analysis were four-fold: (1) to characterize the glycosylation of tralokinumab and the presence of $\alpha$-Gal epitopes in the Fab and Fc domains in vitro; (2) to evaluate the development of tralokinumab Fab-arm exchange and half-antibody formation and their effects on safety and immunogenicity in vitro; (3) to report rates of ADAs in the clinical trials; and (4) to identify possible AEs of anaphylaxis, severe hypersensitivity reactions, and other AEs in the STRATOS 1 and 2 phase III pivotal clinical trials of tralokinumab in participants with severe, uncontrolled asthma.

\section{Methods}

\subsection{Characterization of Glycosylation and the Galactose- $a-1,3-G a l a c t o s e ~(a-G a l)$ Epitope}

Oligosaccharide profile analysis of tralokinumab was conducted to determine the type and abundance of $\mathrm{N}$-linked oligosaccharide species [23]. Release of N-linked glycans from tralokinumab was conducted by incubation with PNGase F (Promega, Madison, WI, USA) at $37^{\circ} \mathrm{C}$ for $16 \mathrm{~h}$. The released $\mathrm{N}$-glycans were then labeled with 2 -aminobenzamide (2-AB). The labeled glycans were cleaned using GlykoClean SPE Cartridges (Prozyme, Hayward, CA, USA). The labeled glycans were separated by hydrophilic interaction liquid chromatography (HILIC) using a BEH $300 \AA 1.7 \mu \mathrm{m}$ glycoprotein column, $2.1 \times 150 \mathrm{~mm}$ (Waters, Taunton, MA, USA) in an ultra-performance liquid chromatography (UPLC) system, eluted with a gradient

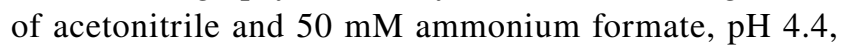
and detected using fluorescence absorbance. The identity of glycan peaks, present at $0.2 \%$ or greater of those detected, was confirmed by in-line electrospray ionization ion-trap mass spectrometry (ESI-MS; Orbitrap Fusion, Thermo Fisher Scientific, Waltham, MA, USA). Identity of 2-AB-labeled glycans was also verified by digestion with exo-glycosidases ( $\alpha$-mannosidase, $\alpha$-galactosidase, $\beta$-galactosidase, and sialidase) at $37{ }^{\circ} \mathrm{C}$ overnight and analyzed by UPLC using the HILIC column already described. The linkages of the terminal monosaccharide units were identified by the changes between the 2-AB oligosaccharide profiles by HILIC before and after digestion with exo-glycosidase.

The methods for determination of the sialic acid content of tralokinumab, characterization of Fab and Fc glycosylation, the reporter gene bioactivity assay, and the neonatal Fc receptor
( $\mathrm{FcRn}$ ) binding assay can be found in Electronic Supplementary Material 1.

\subsection{Half-Antibody Characterization}

Methods for Lys-C peptide mapping to determine tralokinumab disulfide bonds and free thiols, characterization of different levels of reduction of tralokinumab, and Fab-arm exchange with another readily available $\mathrm{IgG}_{4}$ therapeutic $\mathrm{mAb}$ (mavrilimumab) are described in Supplementary Electronic Material 1.

Biological activity of tralokinumab half-antibodies was measured using a human umbilical vein endothelial cells (HUVEC) assay [1], with tralokinumab preventing the IL13-induced expression of vascular cell adhesion molecule-1 (VCAM-1) on the HUVECs. Full details of this methodology have been published previously [1]. HUVECs were obtained from Lonza (Basel, Switzerland) and IL-13 from Peprotech (Rocky Hill, NJ, USA).

Ion exchange chromatography (IEC) fractions enriched with half-antibody were prepared from tralokinumab incubated with PNGase F and carboxypetidase B, and immediately alkylated with $\mathrm{N}$-ethylmaleimide (NEM). Bioactivity of the starting material and the IEC fractions was measured using the HUVEC ELISA (enzyme-linked immunosorbent assay) [1]. $\mathrm{IC}_{50}$ (half-maximal inhibitory concentration) values representing the concentration of tralokinumab at which half-maximal VCAM-1 expression was observed were generated using a four-parameter logistical curve fitted for the tralokinumab reference standard and test samples.

The potency of tralokinumab samples was determined by dividing the $\mathrm{IC}_{50}$ of each test sample by the $\mathrm{IC}_{50}$ of the tralokinumab reference standard and multiplying by 100 .

\subsection{The STRATOS 1 and 2 Phase III Trials}

STRATOS 1 and 2 (ClinicalTrials.gov identifiers NCT02161757 and NCT02194699) were randomized, double-blind, placebo-controlled, parallel-group, phase III clinical trials, which evaluated tralokinumab in participants with severe asthma that was inadequately controlled despite treatment with inhaled corticosteroids plus a long-acting $\beta_{2}$ agonist. Participants aged $12-75$ years received subcutaneous tralokinumab $300 \mathrm{mg}$ every 2 weeks (Q2 W STRATOS 1 and 2), tralokinumab $300 \mathrm{mg}$ every 4 weeks (Q4 W; STRATOS 1 only), or matching placebo. The primary endpoint for both trials was the annualized asthma exacerbation rate reduction at Week $52[6,7]$.

\subsection{Safety Analyses in STRATOS 1 and 2}

All safety summaries and ADA analyses in both trials were based on the safety analysis sets, defined as all participants 
in each trial who received at least one dose of placebo or tralokinumab. In the safety analysis set, participants were assigned to treatment groups based on the actual treatment they received, regardless of which group they were randomized to.

The Medical Dictionary for Regulatory Activities $\left(\right.$ MedDRA $^{\circledR}$ ) version 20.0 was used to code AEs in both trials. AEs in STRATOS 1 were initially coded in MedDRA ${ }^{\circledR}$ version 19.1, but were then recoded in MedDRA ${ }^{\circledR}$ version 20.0 when follow-up data were included.

All AEs were reviewed by study and safety physicians on an ongoing basis. In addition, all safety information was reviewed by an independent Data Safety Monitoring Board.

\subsubsection{Assessment of Anti-Drug Antibodies (ADAs) and Immunogenicity}

Pre-dose serum samples for measuring the presence of ADAs were collected in the STRATOS 1 and 2 trials at baseline (before the first administration of tralokinumab) and at Weeks 26, 56 (6-8 weeks after the final tralokinumab dose), and 72 (22-24 weeks after the final tralokinumab dose). The ADA evaluable population included all participants in the safety population who had non-missing baseline ADA results and at least one non-missing post-baseline ADA result.

The presence and titer of ADAs in serum was detected using a Meso Scale Discovery bridging assay (Meso Scale Discovery, Gaithersburg, MD, USA). Briefly, serum samples were acid dissociated to release any ADAs complexed with free drug. Neutralized samples were mixed with biotinylated tralokinumab and Sulfo-tagged tralokinumab, to allow formation of an antibody-complex bridge. Following incubation, samples were added to a streptavidin-coated plate, and unbound material was washed away. Buffer containing tripolyamine was added, and an electric voltage applied to produce a chemiluminescent signal. Positive results were confirmed by competitive binding with excess tralokinumab to assess the specificity of binding.

For ADA titer determination, the assay was performed using serial dilutions of samples in human serum, with two replicates conducted. The titer was calculated as the reciprocal of the product of the assay's minimum required dilution (1 in 13) multiplied by the greatest serial dilution that yielded a response greater than the assay cut-point. In low concentration samples below the assay cut-point, titer will be reported as $<13$. Consistent with statistical methods mandated by the US Food and Drug Administration (FDA) and the European Medicines Agency (EMA), the cut-point and normalization factor for the ADA screening assay was set to allow a $5 \%$ false-positive rate (determined by a $90 \%$ onesided lower confidence interval [CI] for the 95 th percentile of the negative control population, with a $90 \%$ confidence level) [24, 25]. Similarly, the ADA confirmatory assay cutpoint was set to a $1 \%$ false-positive rate (determined using an $80 \%$ one-sided lower CI for the 99th percentile of the negative control population, with an $80 \%$ confidence level) $[24,25]$.

Confirmed ADA-positive samples were also tested for the presence of neutralizing antibodies (nAbs). Murine antihuman IL-13 mAb, clone 32116, (R\&D Systems, Minneapolis, MN, USA) was immobilized to Meso Scale Discovery High-Bind plates. Samples and controls were incubated in the presence of acidic conditions, and neutralized and incubated in the presence of a known ruthenylated tralokinumab concentration overnight. Recombinant human IL-13 (R\&D Systems, Minneapolis, MN, USA) was added to the blocked assay plates, incubated, and washed to remove unbound material. The sample-tralokinumab complex was then added to the assay plates and unbound material was washed away. Buffer containing tripolyamine was added, and an electric voltage applied to produce a chemiluminescent signal. An inhibition of signal, relative to a non-neutralizing control, was indicative of a neutralizing sample.

Case records of participants with a positive ADA response were reviewed for patterns of AEs.

\subsubsection{Identification of Hypersensitivity Reactions and Possible Anaphylaxis Events}

All AEs of hypersensitivity were identified using the MedDRA ${ }^{\circledR}$ Standardized MedDRA ${ }^{\circledR}$ Query (SMQ) for hypersensitivity, anaphylactic reaction, and anaphylactoid shock conditions. Within this group, a two-step process was used to identify and evaluate a subset comprising possible anaphylactic reactions or any AE for anaphylactoid shock or hypersensitivity. Using a programmatic approach, AEs were identified that occurred either the day of, or within $72 \mathrm{~h}$ of the day after, trial drug administration by a modified algorithm of the SMQ for anaphylactic reaction. The preferred terms (PTs) representing gastrointestinal events were also added to the algorithm. The PTs comprising the algorithm of this SMQ are provided in Table 1. If an AE was captured under the anaphylaxis SMQ, it was automatically considered as one possible anaphylaxis event. If two or more AEs were identified as occurring in two or more of the other categories (for respiratory, dermatological, cardiovascular, and gastrointestinal PTs), these AEs were also considered as one possible anaphylaxis event (Table 1). In addition to this programmatic approach, the blinded study physicians reviewed all AEs that occurred in STRATOS 1 and 2 individually and identified those that could possibly be anaphylaxis events. 


\subsubsection{Evaluation of Possible Anaphylaxis Events}

The clinical evaluation of hypersensitivity reactions for possible anaphylaxis events followed the Sampson criteria [26]. Briefly, the diagnosis of anaphylaxis is highly likely when one of these three clinical scenarios is observed:

1. The acute onset of a reaction (minutes to hours) with involvement of the skin, mucosal tissue, or both, and at least one of the following:

(a) respiratory compromise; or

(b) reduced blood pressure or symptoms of end-organ dysfunction.

2. Two or more of the following occurring rapidly after exposure: involvement of the skin/mucosal tissue, respiratory compromise, reduced blood pressure, or associated symptoms and/or persistent gastrointestinal symptoms.

3. Reduced blood pressure after exposure to an allergen for that participant.

The list of possible anaphylaxis AEs was reviewed by an internal study physician and then referred to an independent, external, blinded evaluator (an allergist who was otherwise not involved in the study) for a participant-level review. The external evaluator was provided with the full profile, a narrative of the event, concomitant medications, medical history, respiratory history, and the dosing data for the participant. The evaluator was asked to determine whether the AE met the Sampson criteria for anaphylaxis. If these criteria were not met, the evaluator was then asked to determine whether the AE represented a non-anaphylactic hypersensitivity event. The evaluator could answer yes, no, or undetermined.

\section{Results}

\subsection{Tralokinumab Glycosylation}

The oligosaccharide profile of tralokinumab can be seen in Fig. 1. The most common oligosaccharides were biantennary glycans with a fucose residue at the core, without a terminal galactose G0f (43.4\%), or with a single terminal galactose $\operatorname{G1f}(\alpha 1,6)(17.1 \%)$ and $\mathrm{G} 1 \mathrm{f}(\alpha 1,3)(14.8 \%)$ (Table 2$)$. Oligosaccharides containing the $\alpha$-Gal epitope were present at $4.5 \%$ in total. Oligosaccharides containing terminal sialic acid residue (NGNA) were present at $10.9 \%$. A high mannose-type oligosaccharide, M5, was present at a very low percentage $(0.6 \%)$.

Fab glycosylation was found to be very low $(<1.0 \%)$ across 21 different manufactured lots of tralokinumab. Separation by size exclusion chromatography (SEC) allowed fractions enriched in glycosylated Fab to be prepared, and its presence was confirmed by intact mass and peptide mapping analysis. Peptide mapping identified two N-glycosylation sites in the heavy chain complementarity-determining region of the Fab, at the asparagine residues 31 and 59. The oligosaccharide profile of the Fab indicated that the major glycans found were sialylated glycans with two or three antennae, core fucosylation, and terminal NGNA residues $\mathrm{G} 2 \mathrm{f}+2 \mathrm{NGc}$ and G3f +3 NGc (Fig. 2). No glycans with $\alpha-G a l$ epitopes were detected in the Fab.

Across tested manufactured lots of tralokinumab covering different processes and clinical stages $(n=19)$, there was some variation in Fc glycosylation, though the proportion of high-mannose and afucosylated species was consistently low at $\leq 1.7 \%$ and $\leq 2.2 \%$, respectively. The proportion of galactosylated species were $\leq 50.8 \%$, species with $\alpha$-Gal epitopes $\leq 9.4 \%$, and sialylated species $\leq 24.1 \%$ of the total Fc glycosylation (see Electronic Supplementary Material 2). Fc deglycosylated tralokinumab had similar bioactivity to control tralokinumab (92\% vs. $100 \%$ respectively), and similar FcRn binding to control tralokinumab ( $87 \%$ vs. $100 \%$, respectively).

\subsection{Tralokinumab Half-Antibody Formation and Fab-Arm Exchange}

A schematic diagram of the disulfide bond pattern of tralokinumab is given in Electronic Supplementary Material 3. The positions of the disulfide bonds were confirmed by comparison of Lys-C peptide maps in non-reducing and reducing liquid chromatography-mass spectrometry (LC-MS) analyses (see Electronic Supplementary Material 4). All domains of tralokinumab had low percentages of free thiols (see Electronic Supplementary Material 5). The heavy chain constant domains had the greatest proportion of free thiols $(2.1-4.1 \%)$, and no free thiols were detected in the hinge region or the heavy chain variable domain. The ratio of free thiols per $\mathrm{mAb}$ molecule for tralokinumab was $0.3 \mathrm{~mol} / \mathrm{mol}$.

Tralokinumab samples were prepared with different degrees of reduction. Figure 3a shows non-reduced tralokinumab separated by non-reducing gel electrophoresis, and mostly comprised tralokinumab monomer (95.2\%). Figure $3 b$ shows reduced tralokinumab; the monomer peak disappeared $(0 \%)$ and was reduced to heavy chains, light chains, and half IgG. In the final sample (Fig. 3c), some of the reduced disulfide bonds had reformed, so that both tralokinumab monomer (30.2\%) and the reduced forms were observed. The proportion of aggregated tralokinumab did not vary a great deal among the reduced samples: $3.0 \%$ (non-reduced), 1.9\% (reduced), and 1.2\% (partially reduced). Reduction of tralokinumab did not appear to have a substantial impact on bioactivity measured by the reporter gene bioassay; bioactivity of the three samples was $94 \%$ 
Table 1 MedDRA ${ }^{\circledR}$ Preferred Terms included in the modified MedDRA ${ }^{\circledR}$ SMQ 'anaphylactic reaction' algorithm ${ }^{\mathrm{a}}$

\begin{tabular}{|c|c|c|c|c|}
\hline Anaphylaxis (A) & Respiratory (B) & Dermatological (C) & Cardiovascular (D) & Gastrointestinal (E) \\
\hline Anaphylactic reaction & Acute respiratory failure & Allergic edema & Blood pressure decreased & Abdominal discomfort \\
\hline Anaphylactic shock & Asthma & Angioedema & $\begin{array}{l}\text { Blood pressure diastolic } \\
\text { decreased }\end{array}$ & Abdominal pain \\
\hline $\begin{array}{l}\text { Anaphylactic transfusion } \\
\text { reaction }\end{array}$ & Bronchial edema & Edema & $\begin{array}{l}\text { Blood pressure systolic } \\
\text { decreased }\end{array}$ & Abdominal pain lower \\
\hline Anaphylactoid reaction & Bronchospasm & Erythema & Cardiac arrest & Abdominal pain upper \\
\hline Anaphylactoid shock & Cardio-respiratory distress & Eye edema & Cardio-respiratory arrest & Gastrointestinal pain \\
\hline Circulatory collapse & Chest discomfort & Eye pruritus & Cardiovascular insufficiency & Vomiting \\
\hline Dialysis membrane reaction & Choking & Eye swelling & Diastolic hypotension & Visceral pain \\
\hline Kounis syndrome & Choking sensation & Eyelid edema & Hypotension & \\
\hline Shock & Circumoral edema & Face edema & & \\
\hline Shock symptom & Cough & Flushing & & \\
\hline \multirow[t]{27}{*}{ Type I hypersensitivity } & Cyanosis & Generalized erythema & & \\
\hline & Dyspnea & Injection-site urticaria & & \\
\hline & Edema mouth & Lip edema & & \\
\hline & Hyperventilation & Lip swelling & & \\
\hline & Irregular breathing & Nodular rash & & \\
\hline & Laryngeal dyspnea & Ocular hyperemia & & \\
\hline & Laryngeal edema & Periorbital edema & & \\
\hline & Laryngotracheal edema & Pruritus & & \\
\hline & Mouth swelling & Pruritus allergic & & \\
\hline & Nasal obstruction & Pruritus generalized & & \\
\hline & Oropharyngeal spasm & Rash & & \\
\hline & Oropharyngeal swelling & Rash erythematous & & \\
\hline & Respiratory arrest & Rash generalized & & \\
\hline & Respiratory distress & Rash pruritic & & \\
\hline & Respiratory failure & Skin swelling & & \\
\hline & Reversible airways obstruction & Swelling & & \\
\hline & Sensation of foreign body & Swelling face & & \\
\hline & Sneezing & Urticaria & & \\
\hline & Stridor & Urticaria papular & & \\
\hline & Swollen tongue & & & \\
\hline & Tachypnea & & & \\
\hline & Throat tightness & & & \\
\hline & Tongue edema & & & \\
\hline & Tracheal obstruction & & & \\
\hline & Tracheal edema & & & \\
\hline & Upper airway obstruction & & & \\
\hline & Wheezing & & & \\
\hline
\end{tabular}

MedDRA Medical Dictionary for Regulatory Activities, SMQ Standardized MedDRA Query

${ }^{a}$ A possible case of anaphylaxis according to the modified MedDRA ${ }^{\circledR}$ algorithm is: any term within category A; or at least two terms within two different categories (or more) within B, C, D, and E

(non-reduced), 91\% (reduced), and 85\% (partially reduced) versus tralokinumab reference standard.

The impact of half-antibody formation on tralokinumab was assessed in samples enriched with half-antibody by IEC fractionation. Analysis of bioactivity using a HUVEC assay demonstrated that the starting material had $119 \%$ bioactivity (vs. tralokinumab reference standard) and 7.5\% half-antibody content. The variation in half-antibody concentration of tralokinumab samples did not impact biological activity. The IEC main peak had $124 \%$ and $0.8 \%$, IEC post-peak 1 had $145 \%$ and $11.9 \%$, and IEC post-peak 2 had $130 \%$ and $65.0 \%$ bioactivity and half-antibody content, respectively. 


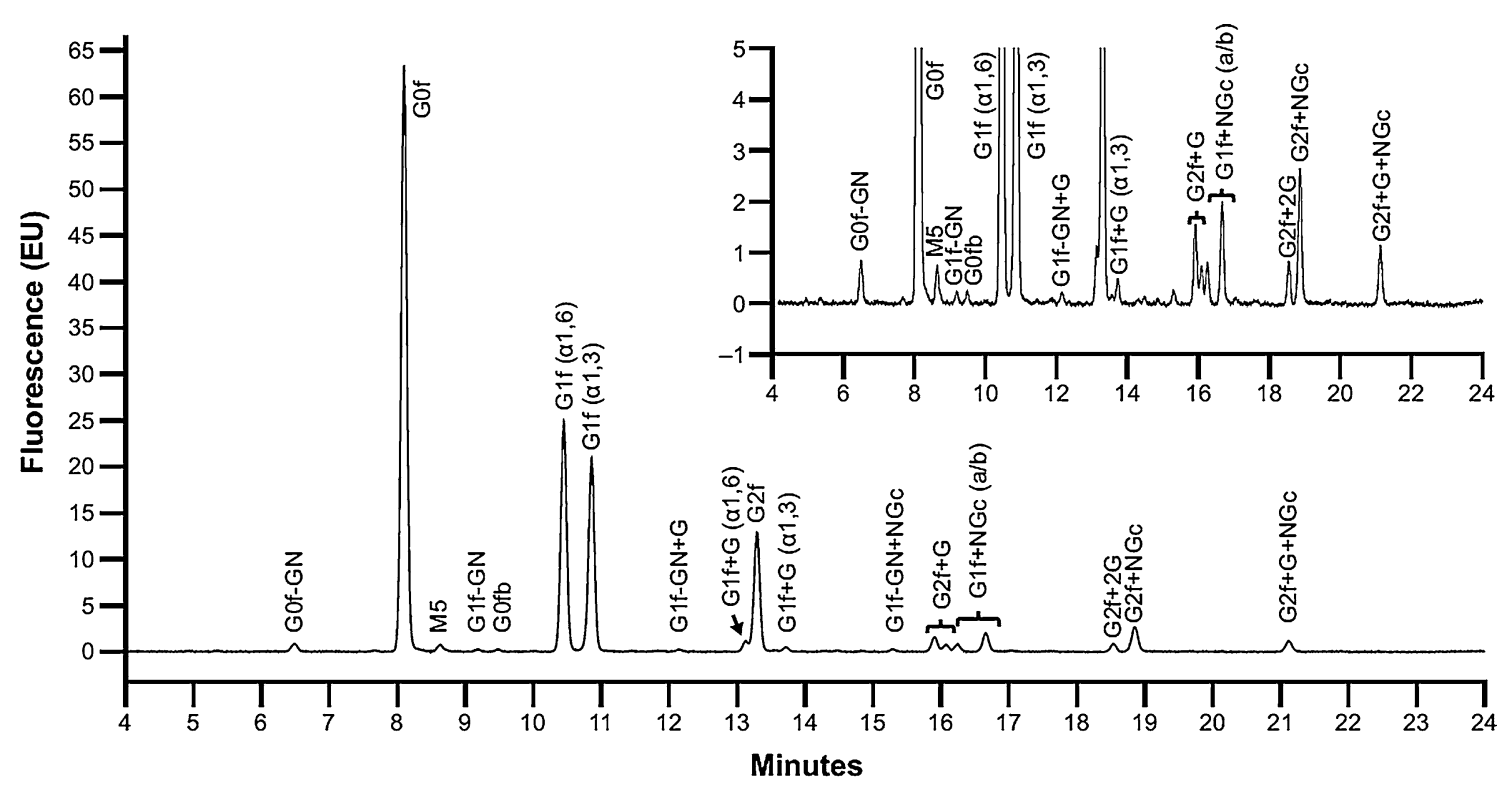

Fig. 1 Oligosaccharide profile of tralokinumab. Separation of 2-aminobenzamide labeled glycans from tralokinumab by hydrophilic interaction liquid chromatography. $f$ fucose, $G$ galactose, $G N$ N-acetyl-glucosamine, $M$ mannose, $N G c$ N-glycolylneuraminic acid

Table 2 Relative percentage of identified oligosaccharides in tralokinumab

\begin{tabular}{lcll}
\hline Oligosaccharide & $\begin{array}{l}\text { Relative } \\
\text { percentage } \\
(\%)\end{array}$ & $\begin{array}{l}\text { Theoretical } \\
\text { mass (Da) }\end{array}$ & Observed mass (Da) \\
\hline Complex type & & 1582.61 & 1582.65 \\
G0f & 43.4 & 1744.67 & 1744.71 \\
G1f $(\alpha 1,6)$ & 17.1 & 1745.67 & 1745.70 \\
G1f $(\alpha 1,3)$ & 14.8 & 1906.72 & 1906.76 \\
G2f & 8.8 & 2051.71 & 2051.81 \\
G1f + NGc (a/b) & 4.7 & 2213.76 & 2213.86 \\
G2f + NGc & 4.2 & 2375.82 & 2375.92 \\
G2f $+G+N G c$ & 1.6 & 2068.77 & 2068.82 \\
G2f $+G$ & 1.1 & 1379.53 & 1379.57 \\
G0f-GN & 0.7 & 1906.72 & 1906.76 \\
G1f $(\alpha 1,6)+G$ & 0.7 & 2230.83 & 2230.88 \\
G2f $+2 G$ & 0.5 & 1906.72 & 1906.76 \\
G1f( $\alpha 1,3)+G$ & 0.4 & 1848.63 & 1848.72 \\
G1f-GN + NGc & 0.4 & 1541.59 & 1541.62 \\
G1f-GN $(\mathrm{a} / \mathrm{b})$ & 0.2 & 1703.64 & 1703.68 \\
G1f-GN $+G$ & 0.2 & 1785.69 & 1785.73 \\
G0fb & 0.1 & 1354.50 & 1354.53 \\
High-mannose type & & & \\
M5 & 0.6 & & \\
\hline
\end{tabular}

Oligosaccharides containing the galactose- $\alpha$-1,3-galactose ( $\alpha$-Gal) epitope are indicated in italics

$f$ fucose, $G$ galactose, $G N$ N-acetyl-glucosamine, $M$ mannose, $N G c$ $N$-glycolylneuraminic acid

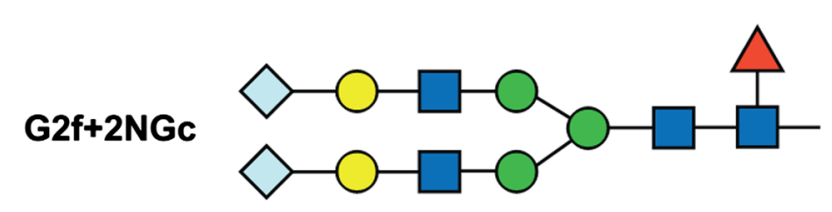

G3f+3NGc

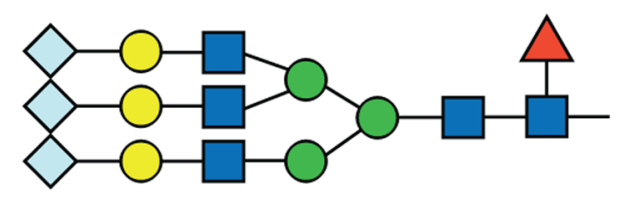

Man $\square$ GIcNAc $\triangle$ Fuc $O$ Gal $\diamond N G N A$

Fig. 2 Structure and nomenclature of oligosaccharides in the tralokinumab Fab region. $f$ fucose, $G$ galactose, GlcNAc N-acetylglucosamine, Man mannose, $N G c$ N-glycolylneuraminic acid

Tralokinumab incubated in IgG-depleted human serum at $37{ }^{\circ} \mathrm{C}$ for 8 weeks had $106 \%$ bioactivity at Week 0 (vs. tralokinumab reference standard), which decreased during the incubation to $70 \%$ at Week 8 . The amount of halfantibody was $5.5 \%$ at Week 0 and increasing proportions of half-antibody were detected during the incubation period, varying from 10.6 to $18.5 \%$. The percentage of monomer as measured by SEC was $74.9 \%$ at Week 8 . This indicated 
that the decrease in bioactivity was probably caused by other modifications such as aggregation and fragmentation. Bioactivity results indicated that the in vitro half-antibody had similar activity to whole tralokinumab.

Tralokinumab did not readily undergo Fab-arm exchange with mavrilimumab under physiological conditions in vitro. Following incubation of non-reduced or reduced tralokinumab with non-reduced mavrilimumab in IgG-depleted human serum, no Fab-arm exchanged hybrid antibody was observed in incubates separated by IEC (Fig. 4a). In contrast, hybrid antibody was detected by IEC when both tralokinumab and mavrilimumab were reduced with glutathione before incubation (Fig. 4b). The identity of the hybrid antibody was confirmed by intact mass analysis. Bioactivity of the hybrid antibody was expected to be $50 \%$ of intact tralokinumab; the measured bioactivity (40\%) of the hybrid molecule was close to the expected theoretical level.

\subsection{Safety Within the STRATOS 1 and 2 Clinical Trials}

\subsubsection{Exposure}

A total of 1227 participants received tralokinumab in the STRATOS 1 and 2 trials (Fig. 5). In STRATOS 1, 398 participants received tralokinumab Q2 W and 404 participants received tralokinumab Q4 W. In STRATOS 2, 425 participants received tralokinumab Q2 W [7]. The combined duration of exposure across both trials was 409,595 days (Q2 W); 267,698 days (Q4 W) for STRATOS 1 and 141,897 days for STRATOS 2.
Fig. 3 Characterization of tralokinumab with different degrees of reduction by nonreducing gel electrophoresis: (a) non-reduced; (b) reduced; and (c) partially reduced. $H H L$ two heavy chains + one light chain, Ig $G$ immunoglobulin $\mathrm{G}$
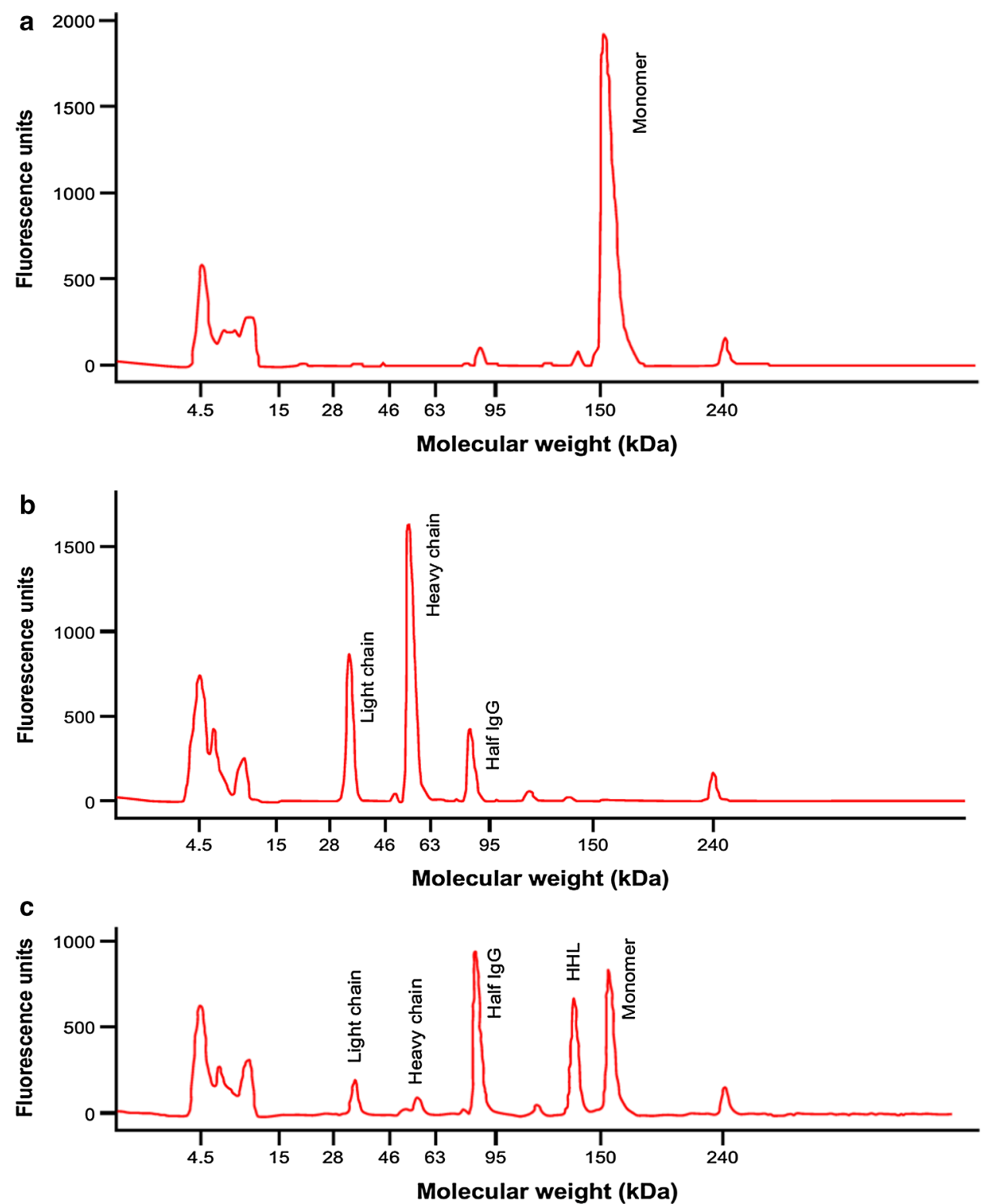


\subsubsection{Adverse Events}

The most frequent AEs in STRATOS 1 and STRATOS 2 (reported by $\geq 5 \%$ of participants) were asthma, headache, and upper respiratory tract infection [7]. Review of all AEs revealed that the safety profile of tralokinumab was consistent with that observed in previous trials [7].

\subsubsection{ADA Assessment}

Within STRATOS 1 there was no obvious relationship between treatment group and either ADA prevalence or incidence [7]. The ADA prevalence, i.e., the proportion of the study population having drug-reactive antibodies at any point in time, was 7/398 (1.9\%) in the tralokinumab Q2 W group, 7/404 (1.8\%) in the tralokinumab Q4 W group, and $9 / 400(2.4 \%)$ in the placebo group.

Of the seven ADA-positive participants in the tralokinumab Q2 W group, four had pre-existing ADA (ADA-positive at baseline) and three had a positive result post-baseline not detectable at baseline. Of these three, two were persistently ADA-positive (with $\geq 2$ positive post-baseline assessments that had either $\geq 16$ weeks between the first and last positive assessment, or were positive at the last assessment); one was transiently ADA-positive (with $\geq 1$ positive postbaseline assessment, but not fulfilling the persistent definition). Of the seven ADA-positive participants in the tralokinumab Q4 W group, five had pre-existing ADA and two had a positive result post-baseline not detectable at baseline. In total, three participants were positive post-baseline (one of whom had pre-existing ADAs); of these, two were persistently ADA-positive and one was transiently ADA-positive. Of the nine ADA-positive participants in the placebo group, seven had pre-existing ADA and two had a positive result post-baseline not detectable at baseline. Eight participants were positive post-baseline (six of whom had pre-existing ADAs); of these, seven were persistently ADA-positive and one was transiently ADA-positive. No participants in either tralokinumab group had treatment-boosted ADA (defined as baseline positive ADA titer that was boosted to a four-fold or greater level following drug administration). One ( $0.3 \%)$ participant met this criterion in the placebo group. Thus, the ADA incidence (defined as the sum of both treatmentinduced [post-baseline ADA-positive only] and treatmentboosted ADA) was $0.8 \%$ (3/373) in the tralokinumab Q2 W group, $0.5 \%(2 / 382)$ in the tralokinumab $\mathrm{Q} 4 \mathrm{~W}$ group, and
Fig. 4 Ion exchange chromatography profiles of tralokinumab and mavrilimumab Fab-arm exchange under (a) physiological conditions and (b) non-physiological conditions
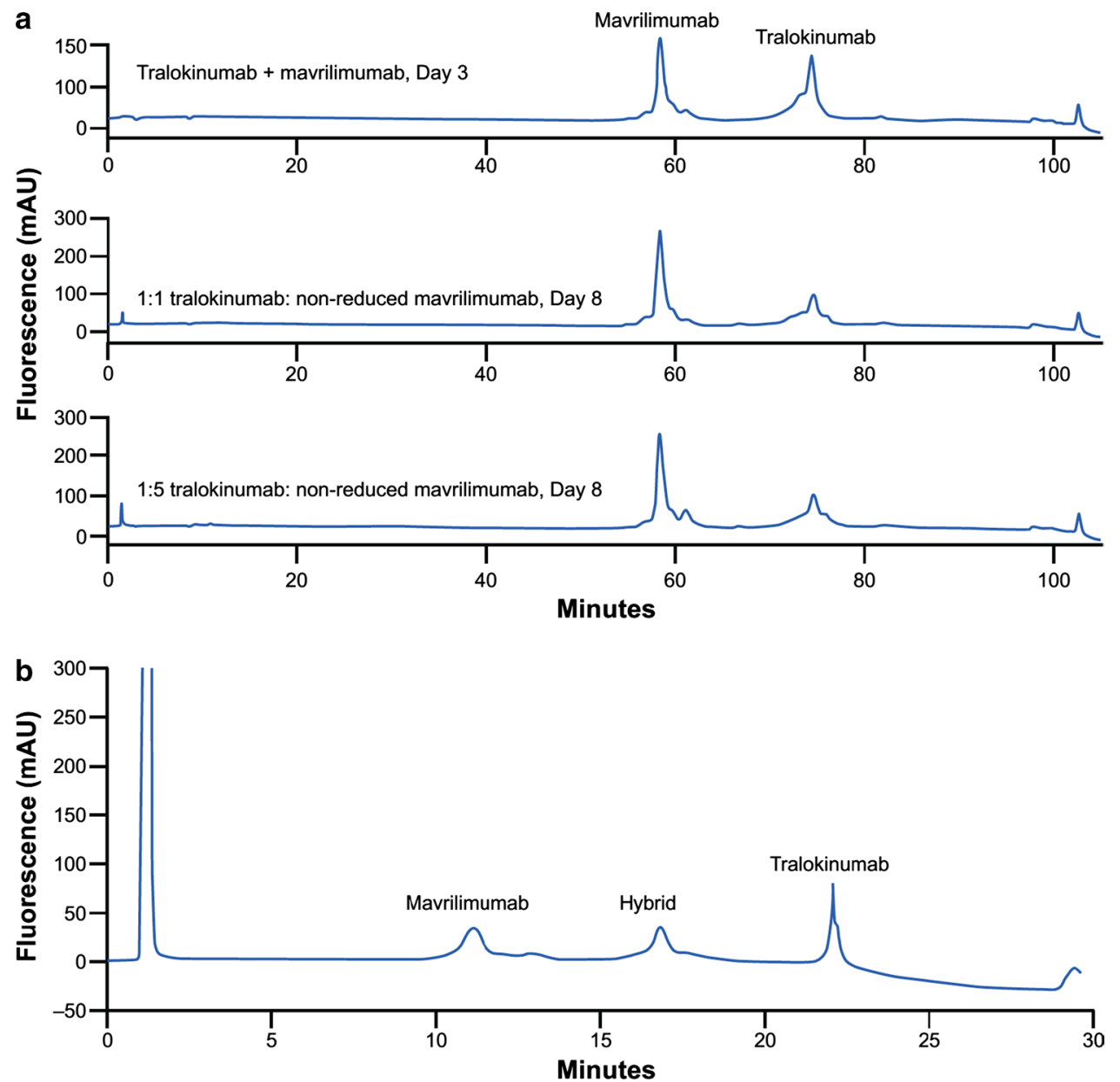

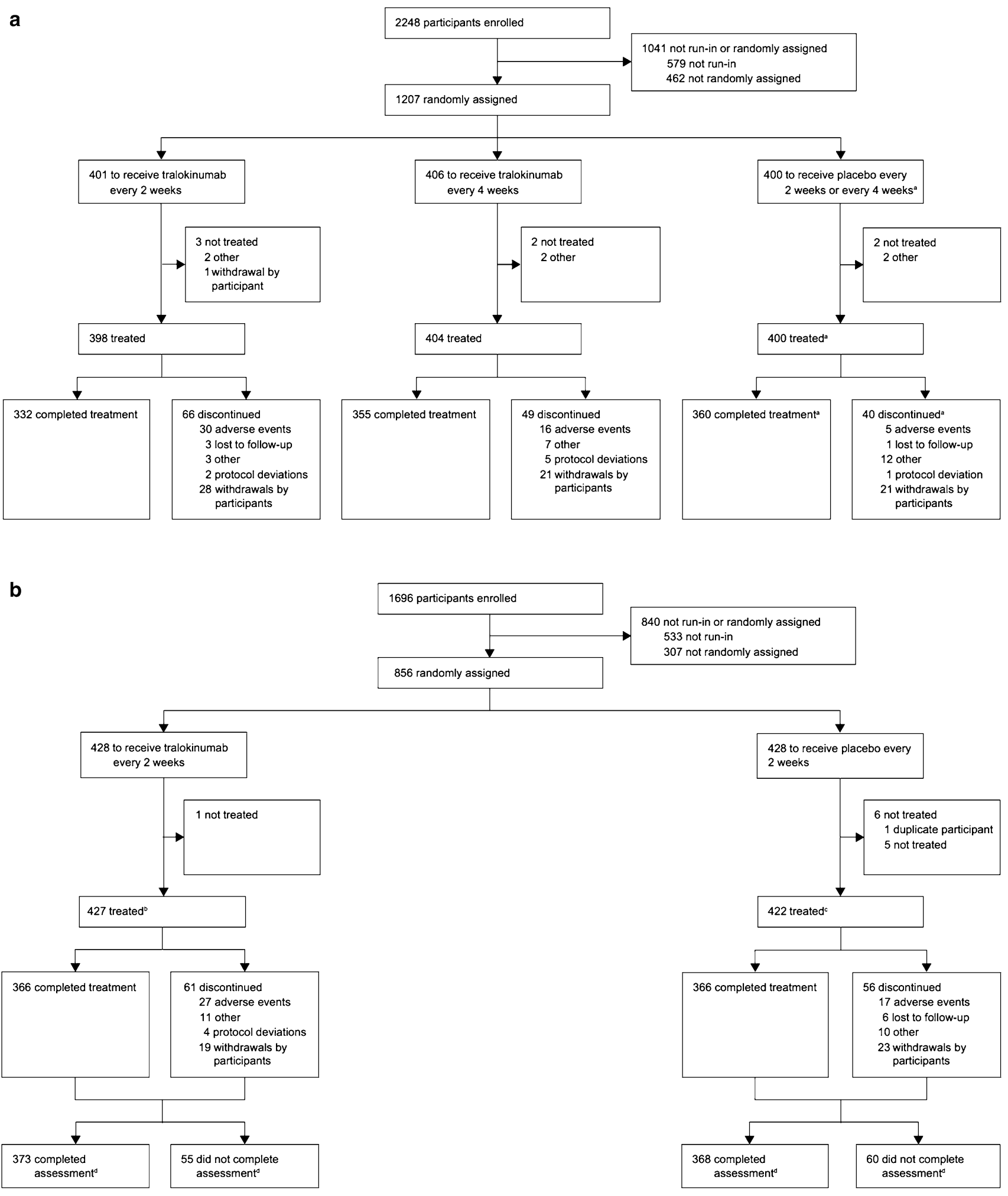

Fig. 5 Patient flow diagram for (a) STRATOS 1 and (b) STRATOS 2. ${ }^{a}$ The STRATOS 1 placebo treatment group is a combined treatment group (placebo every 2 weeks plus placebo every 4 weeks), where the two placebo cohorts were given weights proportional to the number of participants in each cohort. For STRATOS 2, eight participants on tralokinumab every 2 weeks and 11 on placebo were excluded from the full analysis set. ${ }^{\mathrm{b}}$ Two participants were duplicates and were not included in the safety set; these two participants and five who did not have the potential to receive 52 weeks of treatment were not included in the full analysis set. ${ }^{c}$ Five participants did not have the potential to receive 52 weeks of treatment, so were not included in the full analysis set. ${ }^{\mathrm{d}}$ Of participants randomly assigned. Republished with permission of Elsevier, from Panettieri et al. [7]; permission conveyed through the Copyright Clearance Center, Inc 
$0.8 \%(3 / 374)$ in the placebo group. ADA titers were low in nature, with median (range) titers of $26.0(<13-26), 26.0$ (26-26), and 52.0 (13-208), respectively. See Electronic Supplementary Material 1 for titers for participants with persistent and transient positive post-baseline ADAs. Five (1.3\%) participants in each of the tralokinumab groups and four $(1.1 \%)$ participants in the placebo group tested positive for the presence of nAbs against tralokinumab.

Within STRATOS 2, again there was no obvious relationship between either ADA prevalence or incidence and treatment group. The ADA prevalence was $7 / 405(1.7 \%)$ in the tralokinumab Q2 W group and 8/394 (2.0\%) among placebo recipients.

Of the seven ADA-positive participants in the tralokinumab Q2 W group, five had a positive result post-baseline not detectable at baseline (three were persistently ADA-positive and two were transiently ADA-positive) and two had pre-existing ADA. For the eight ADA-positive participants in the placebo group, six had a positive result post-baseline (all six were persistently ADA-positive; three also had preexisting ADAs and three had a positive result post-baseline not detectable at baseline), and two who had pre-existing ADA only. No participants in the tralokinumab or the placebo group had treatment-boosted ADA. Thus, the ADA incidence was $1.2 \%$ (5/405 participants) for the tralokinumab Q2 W group compared with $0.8 \%$ (3/394) for placebo. ADA titers were low in nature, with median (range) titers of 39.0 $(<13-208)$ and 13.0 (13-26), respectively. See Electronic Supplementary Material 1 for titers for participants with persistent and transient positive post-baseline ADAs. All five of the ADA-positive participants in the tralokinumab Q2 W group tested positive for the presence of nAb against tralokinumab. However, it should be noted that the number of ADA-positive participants in each of the treatment groups was small (less than ten), and thus it is not possible to draw definitive conclusions.

A review of AEs in the ADA-positive participants indicated that the safety profile of tralokinumab was consistent with that observed in previous trials; however, the numbers were too small for any meaningful interpretation of patterns of AEs. See Electronic Supplementary Material 6 for the most frequently reported AEs in ADA-positive participants.

\subsubsection{Anaphylaxis}

Analysis of MedDRA ${ }^{\circledR}$ SMQs during the treatment period identified AEs of hypersensitivity, anaphylactoid shock, and anaphylaxis. In STRATOS 1, hypersensitivity AEs were reported by 103/398 participants (25.9\%) in the tralokinumab Q2 W group, 101/404 (25.0\%) in the tralokinumab Q4 W group, and 102/400 (25.5\%) in the placebo group. In STRATOS 2, hypersensitivity AEs were reported by $56 / 425$ participants $(13.2 \%)$ in the tralokinumab
Q2 W group and 38/422 (9.0\%) in the placebo group (see Electronic Supplementary Material 7). No cases of hypersensitivity type III reactions were reported in either STRATOS 1 or 2.

No cases of potential hypersensitivity reactions related to tralokinumab administration met the Sampson criteria for anaphylaxis in either STRATOS 1 or STRATOS 2 (see Electronic Supplementary Material 8). In STRATOS 1, one patient reported pharyngeal edema and a swollen tongue, which were considered to be related to tralokinumab administration, but did not meet Sampson criteria for anaphylaxis (see Electronic Supplementary Material 8). Conversely, the criteria were met by two AEs (urticaria and wheezing) in a participant receiving placebo in STRATOS 2. One AE (urticaria) in a participant in the tralokinumab Q2 W group of STRATOS 2 met the Sampson criteria for non-anaphylactic hypersensitivity.

\section{Discussion}

In this study, characterization of the glycans of tralokinumab and its ability to undergo Fab-arm exchange did not suggest that there would be an increased risk for anaphylaxis or hypersensitivity reactions with tralokinumab treatment. The clinical safety data from the STRATOS 1 and 2 trials reported here were in line with these in vitro data, with low reported incidence of ADAs and nAbs, and no anaphylaxis or severe hypersensitivity reactions according to Sampson criteria.

In vitro analyses determined that small amounts of tralokinumab contained glycoforms with the $\alpha$-Gal epitope, which were only detected in the Fc region and not expected to impact tralokinumab immunogenicity. Although therapeutic mAbs often contain detectable concentrations of $\alpha-\mathrm{Gal}$ epitopes, studies have demonstrated that binding by $\operatorname{IgE}$ antibodies appears to be restricted to $\alpha$-Gal epitopes in the Fab domains only $[10,27]$. It is thought this is the result of steric hindrance in the $\mathrm{Fc}$ domain that prevents IgE binding to the $\alpha$-Gal epitopes [28]. In contrast, cetuximab (chimeric $\mathrm{IgG}_{1}$ epidermal growth factor receptor [EGF-R] $\mathrm{mAb}$ ) is $\mathrm{N}$-glycosylated in both the $\mathrm{Fab}$ and $\mathrm{Fc}$ regions; in the $\mathrm{V}_{\mathrm{H}}$ and $\mathrm{C}_{\mathrm{H}} 1$ domain of the Fab region, $89 \%$ of glycans contain $\alpha$-Gal epitopes while $\alpha$-Gal epitopes are undetectable in the Fc region [29]. While cetuximab and tralokinumab were both produced in murine cell lines that are known to introduce $\alpha$-Gal epitopes ( $\mathrm{Sp} 2 / 0$ and NS0, respectively), the amount and distribution of $\alpha-G a l$ epitopes were different in the two mAbs.

Tralokinumab was found to contain glycans with the sialylated species N-glycolylneuraminic acid (NGNA). Although anti-NGNA antibodies have been reported in humans [30, 31], no ADAs associated with NGNA have 
been reported in therapeutic antibodies to date, so the presence of NGNA is not thought to pose a concern for the immunogenicity of tralokinumab. The presence of highmannose glycans in antibodies is associated with enhanced antibody-dependent cell-mediated cytotoxicity (ADCC), decreased complement-dependent cytotoxicity (CDC), and may lead to more rapid clearance in serum [32-36], which could affect the antibodies' bioactivity. Moreover, several studies in mice have shown that the immunogenicity of recombinant proteins with different glycosylation patterns is impacted by the presence of mannose-containing glycans [37-39]. However, ADCC and CDC are not thought to form part of the mechanism of action of tralokinumab, and we have demonstrated that the deglycosylated $\mathrm{Fc}$ had similar relative bioactivity and FcRn binding as the glycosylated control. In addition, we observed low concentrations of high-mannose glycans in the samples in this study. Overall, this suggests that high-mannose glycans are unlikely to influence tralokinumab bioactivity or immunogenicity, and that variations in $\mathrm{Fc}$ glycoforms would be unlikely to impact the pharmacokinetics or pharmacodynamics of tralokinumab.

Using non-reducing Lys-C peptide mapping, we confirmed that the disulfide bond structure of tralokinumab is consistent with the typical structure for native and recombinant $\mathrm{IgG}_{4}$ antibodies: four inter-chain disulfide bonds, of which two were at the hinge region connecting the heavy chains and 12 intra-chain disulfide bonds [21]. Reduction of tralokinumab's disulfide bonds did not appear to have a substantial impact on its bioactivity. The percentage of free thiol groups detected in tralokinumab was low $(0.3 \mathrm{~mol} /$ mol free thiol to $\mathrm{mAb}$ ), suggesting the likelihood of intermolecular disulfide bond formation using free thiol groups would be low. IgG antibodies with more free thiols are also more likely to have exposed hydrophobic regions, which can form aggregates through hydrophobic interactions between molecules [21]. Phagocytic cell removal of these antibody aggregates can subsequently elicit an immune response [40]. However, partially reduced tralokinumab samples did not show increased aggregation (as measured by SEC compared with non-reduced tralokinumab), suggesting that the impact of tralokinumab's partial reduction on immunogenicity is low.

In vitro analyses of samples in which half-antibody was enriched by IEC fractionation showed that the concentration of half-antibody did not affect the biological activity of tralokinumab. Further, in this study, tralokinumab did not readily undergo Fab-arm exchange in vitro under physiological, non-reducing conditions when tested with mavrilimumab. This was consistent with previous observations in vitro, in which $\mathrm{IgG}_{4}$ was reported to have strong non-covalent interactions between $\mathrm{C}_{\mathrm{H}} 3$ domains and formed a stable four-chain molecule [18]. In contrast, when both tralokinumab and mavrilimumab were fully reduced, we observed that a hybrid antibody was formed whose bioactivity was close to predicted levels.

Fab-arm exchange is an inherent characteristic of wildtype $\mathrm{IgG}_{4}$ antibodies [19, 20, 41], and has been observed in vivo with therapeutic mAbs [18-20] and in pathogenic autoantibodies [42]. It has been observed, both in mice and humans, that Fab-arm exchange in $\mathrm{IgG}_{4}$ antibodies is dependent on the amino acid sequence in the core-hinge area [19]. Half-antibody formation and Fab-arm exchange with endogenous $\operatorname{IgG}_{4}$ is therefore not expected to impact on the safety profile of tralokinumab. It has been hypothesized that the formation of bispecific antibodies with endogenous $\mathrm{IgG}_{4}$ antibodies may lead to undesired cross-linking resulting in severe AEs [14, 19, 20], but thus far there have been no reports directly linking Fab-arm exchange in $\mathrm{IgG}_{4}$ therapeutic $m A$ Abs with AEs in treated individuals [43]. In a simple pharmacokinetic model developed from experimental data, Labrijn et al. [19] showed that under normal serum conditions, the majority of therapeutic $\mathrm{IgG}_{4}$ is expected to be monovalent bispecific antibody, which serves as a natural buffer to substantially reduce undesired cross-linking by bispecific antibodies.

The ADA incidence, prevalence and titers in STRATOS 1 and 2 were similar between the tralokinumab and placebo groups. Compared with placebo, there was no evidence of treatment-boosted ADA titers in patients who received repeated dosages of tralokinumab. The number of participants with post-baseline ADAs and nAbs was small (less than ten). Given the low reported rate of ADAs and difficulty in predicting individual patients' immune responses, identification of specific properties that contributed to the immunogenicity of tralokinumab is difficult, and would be purely speculative without further investigation. Indeed, it is possible that the development of ADAs was not related to the therapeutic effects of tralokinumab. However, the participants with ADA-positive samples did not appear to have reduced tralokinumab exposure. Potentially, therefore, these samples categorized as positive were false-positive results, consistent with the fixed 5\% false-positive rate of the ADA screening assay and $1 \%$ false-positive rate of the confirmatory assay. In support of this, no pattern of AEs was detected in ADA-positive participants in the current analysis.

In the STRATOS 1 and 2 trials, the safety profile of tralokinumab was consistent with that observed in previous trials. Hypersensitivity AEs were reported by similar numbers of participants in the tralokinumab and placebo treatment groups in both studies. None of the reported hypersensitivity reactions were type III, which are known to result from the presence of ADAs [22]. While asthma is a known risk factor for anaphylaxis [44, 45], no hypersensitivity or anaphylactic reactions were identified according to the blinded, external assessment using the Sampson criteria in tralokinumab-treated participants in 
either trial. A recent trial of lebrikizumab, another $\operatorname{IgG}_{4}$ $\mathrm{mAb}$ administered subcutaneously, also reported small numbers of hypersensitivity reactions in clinical use in asthma [46].

Our clinical observations with tralokinumab can be contrasted with cetuximab, with which a high prevalence of hypersensitivity reactions (bronchospasm, stridor, hoarseness, urticaria, and/or hypotension) was noted during clinical trials of colorectal cancer and squamous cell carcinoma of the head and neck in the USA. In Tennessee and North Carolina, $22 \%$ of patients treated with cetuximab had severe hypersensitivity reactions, while rates were lower $(<1 \%)$ in most centers in the Northeast of the country [47, 48]. Among those treated with cetuximab in Southeast USA, most had $\mathrm{IgE}$ antibodies specific for $\alpha$-Gal. It was suggested that the regional difference observed might be due to exposure to regional pests and diseases, such as ticks [10]. As a result, cetuximab has a boxed warning for infusion-related, including anaphylactic, reactions in the US label [12]. Mild or moderate infusion-related reactions are listed as very common $(\geq 1 / 10)$ and severe infusion-related reactions, sometimes with fatal outcomes, are listed as common ( $\geq 1 / 100$ to $<1 / 10$ ) in the European Union (EU) Summary of Product Characteristics (SmPC). Anaphylactic reactions may occur in the first infusion or with subsequent infusions and the risk increases with a history of allergy to red meat or tick bites, or presence of anti- $\alpha$-Gal IgE antibodies [49]. The US label notes that severe infusion reactions, sometimes fatal, may occur in about $3 \%$ of patients [12].

With other therapeutic mAbs produced in murine cell lines that are administered subcutaneously, rates of hypersensitivity reactions and anaphylaxis were generally low, and it is important to note that manufacture of a mAb in a murine cell line is not a predictor of increased risk of severe hypersensitivity reactions in humans. For example, golimumab (human $\mathrm{IgG}_{1} \mathrm{~K}$ anti-tumor necrosis factor [TNF]- $\alpha \mathrm{mAb}$ ), ustekinumab (human $\mathrm{IgG}_{1} \kappa \mathrm{mAb}$ targeting the $\mathrm{p}-40$ subunit of $\mathrm{IL}-12$ and IL-23), and canakinumab (human $\operatorname{IgG}_{1} \kappa$ anti-IL-1 $\beta$ mAb) are all produced in the same murine cell line as cetuximab, Sp2/0, and daclizumab (humanized $\mathrm{IgG}_{1}$ anti-IL-2R $\alpha \mathrm{mAb}$ ) is produced in murine cell line NS0, but none are associated with hypersensitivity reactions or anaphylaxis at the same frequency [50-53].

Of 1659 golimumab-treated participants from five phase II and III trials in rheumatoid arthritis (RA), psoriatic arthritis (PsA), and ankylosing spondylitis, none developed anaphylactic reactions [50, 54]. However, in post-marketing experience, serious systemic hypersensitivity reactions, including anaphylaxis, have been reported, of which some reactions occurred after the first administration of golimumab. The golimumab SmPC lists allergic reactions (bronchospasm, hypersensitivity, urticaria) as common $(\geq 1 / 100$ to $<1 / 10)$, and serious systemic hypersensitivity reactions (including anaphylactic reactions) as rare $(\geq 1 / 10,000$ to $<1 / 1000)$ [50]. Similarly, clinical trials (involving 4135 participants with psoriasis and/or PsA who received active drug) and postmarketing studies of ustekinumab have reported hypersensitivity reactions as uncommon $(\geq 1 / 1000$ to $<1 / 100)$ and serious hypersensitivity reactions, including anaphylaxis and angioedema, as rare $(\geq 1 / 10,000$ to $<1 / 1000)$ [52]. For canakinumab, no anaphylactic or anaphylactoid reactions were reported in clinical trials in gouty arthritis $(n=691)$ and RA $(n=441)$ [55]. Hypersensitivity reactions associated with canakinumab treatment have been reported, though the symptoms of cryopyrin-associated periodic syndromes for which canakinumab is approved are similar to symptoms of hypersensitivity [53]. The daclizumab US label has a warning for anaphylaxis and angioedema and notes that anaphylaxis, angioedema, and urticaria can occur after the first dose or at any time during treatment. Multiorgan hypersensitivity was observed in two or more patients in trials treating relapsing multiple sclerosis [51].

The low ADA incidence, similar frequency of hypersensitivity reactions in tralokinumab- and placebotreated participants, and lack of Sampson criteria-defined anaphylaxis in the STRATOS 1 and 2 clinical trials support our assessment that tralokinumab is unlikely to be associated with an increased risk of severe hypersensitivity reactions based on in vitro characterization of the antibody structure. This favorable immunological safety profile is expected to extend to people with atopic dermatitis treated with tralokinumab, for which phase III trials are currently ongoing (NCT03131648; NCT03160885).

\subsection{Limitations}

The statistical methods used in the determination of ADA titers, as mandated in the US FDA and EMA guidance documents [24, 25], may have resulted in biased estimates of the screening, confirmatory, and neutralizing cut-points established from the study populations. This could cause the false-positive rate to be greater than expected [56]. Further, some of the analyses were undertaken in the in vitro setting. As a result, the findings may not be truly representative of the complex in vivo situation and, thus, have limited applicability in the clinical setting [57].

\section{Conclusions}

In vitro analyses and safety data from two pivotal phase III clinical trials of people with severe, uncontrolled asthma have not identified an increased risk of severe hypersensitivity or anaphylactic reactions with 
tralokinumab. In vitro characterization of tralokinumab's glycans found that the $\alpha-G a l$ epitope was present in small amounts in the $\mathrm{Fc}$ region only. The half-antibody content of tralokinumab did not appear to affect bioactivity and tralokinumab did not undergo Fab-arm exchange easily in vitro. The rates of ADAs in the STRATOS 1 and 2 trials were low. No pattern of other AEs was found in ADApositive participants. Together these results suggest that tralokinumab treatment would not lead to an increased risk for severe hypersensitivity or anaphylactic reactions.

Acknowledgements The authors thank the healthcare providers, research staff, participants, and caregivers who participated in the STRATOS 1 and 2 trials. They would also like to thank Klas Lindell for the help of identifying possible cases of severe hypersensitivity reactions and anaphylaxis (Patient Safety, AstraZeneca, Gothenburg, Sweden). Medical writing support was provided by Sally Cotterill, PhD (QXV Comms [Macclesfield, UK], an Ashfield Company, part of UDG Healthcare plc), funded by AstraZeneca (Cambridge, UK), in accordance with Good Publication Practice (GPP3) guidelines (http:// www.ismpp.org/gpp3).

Author Contributions Mats Carlsson contributed to the design of the STRATOS trials, and collection, analysis, and interpretation of clinical safety data from the STRATOS trials. Martin Braddock contributed to the analysis and interpretation of the in vitro and preclinical studies and building the scientific link with the clinical safety data from the STRATOS trials. Yuling Li led team efforts on developing the production process and analytical methods for tralokinumab. Jihong Wang contributed to the design, execution, data analysis, and interpretation of the in vitro experiments. Weichen Xu contributed to the design, execution, data analysis, and interpretation of the in vitro experiments. Nicholas White developed, validated, and oversaw the ADA bioanalysis of the ATMOSPHERE program, including the STRATOS trials; contributed to protocol design, for the ADA strategy; and was an author of the Clinical Study Report for both STRATOS trials. Ayman Megally contributed to the design, execution, and data analysis of the reporting system for hypersensitivity and anaphylaxis reactions to the external evaluator, and performed the role of study physician during both STRATOS trials. Gillian Hunter contributed to the analysis and interpretation of clinical data from the STRATOS trials. Gene Colice contributed to the design of the STRATOS trials, and collection, analysis, and interpretation of clinical safety data from the STRATOS trials.

\section{Compliance with Ethical Standards}

Funding This study was sponsored by AstraZeneca. AstraZeneca was involved in the study design, analysis, and interpretation of the data, and in the writing of this manuscript. Data underlying the findings described in this manuscript may be obtained in accordance with AstraZeneca's data sharing policy described at https://astrazenecagrouptria ls.pharmacm.com/ST/Submission/Disclosure.

Conflicts of interest Yuling Li was an employee of MedImmune, the biologics division of AstraZeneca, at the time the study took place; her current affiliation is CBT Pharmaceuticals. Jihong Wang and Weichen $\mathrm{Xu}$ are employees of MedImmune, the biologics division of AstraZeneca. Nicholas White is an employee of MedImmune and an AstraZeneca shareholder. Mats Carlsson, Martin Braddock, and Ayman Megally are employees of AstraZeneca, the sponsor of STRATOS 1 and STRATOS 2. Gene Colice is an employee of and holds stock options in AstraZeneca. Gillian Hunter is a consultant employed by AstraZeneca.
Ethical approval and informed consent STRATOS 1 and STRATOS 2 were performed in accordance with the ethical principles that have their origin in the Declaration of Helsinki and that are consistent with International Conference on Harmonisation (ICH)/Good Clinical Practice (GCP), applicable regulatory requirements, and the AstraZeneca policy on Bioethics. All participating centers obtained approval from an independent ethics committee and all participants provided written informed consent.

OpenAccess This article is distributed under the terms of the Creative Commons Attribution-NonCommercial 4.0 International License (http://creativecommons.org/licenses/by-nc/4.0/), which permits any noncommercial use, distribution, and reproduction in any medium, provided you give appropriate credit to the original author(s) and the source, provide a link to the Creative Commons license, and indicate if changes were made.

\section{References}

1. May RD, Monk PD, Cohen ES, Manuel D, Dempsey F, Davis NH, et al. Preclinical development of CAT-354, an IL-13 neutralizing antibody, for the treatment of severe uncontrolled asthma. Br J Pharmacol. 2012;166(1):177-93.

2. Popovic B, Breed J, Rees DG, Gardender MJ, Vinall LMK, Kemp B, et al. Structural characterisation reveals mechanism of IL-13 neutralising monoclonal antibody tralokinumab as inhibition of binding to IL-13R $\alpha 1$ and IL-13R $\alpha 2$. J Mol Biol. 2017;429:208-19.

3. Corren J. Role of interleukin-13 in asthma. Curr Allergy Asthma Rep. 2013;13(5):415-20.

4. Wills-Karp M. Interleukin-13 in asthma pathogenesis. Curr Allergy Asthma Rep. 2004;4(2):123-31.

5. Panettieri R, Wang M, Braddock M, Bowen K, Colice G. Tralokinumab for the treatment of severe, uncontrolled asthma: the ATMOSPHERE clinical development program. Immunotherapy. 2018;10(6):473-90.

6. Panettieri RA Jr, Brightling C, Sjobring U, Péterffy A, Tornling G, Daoud SZ, et al. STRATOS 1 and 2: considerations in clinical trial design for a fully human monoclonal antibody in severe asthma. Clin Invest (Lond). 2015;5(8):701-11.

7. Panettieri R, Sjobring U, Péterffy A, Wessman P, Bowen K, Piper E, et al. Tralokinumab for severe, uncontrolled asthma (STRATOS 1 and STRATOS 2): two randomised, double-blind, placebo-controlled, phase 3 clinical trials. Lancet Respir Med. 2018;6(7):511-25.

8. Harding FA, Stickler MM, Razo J, DuBridge RB. The immunogenicity of humanized and fully human antibodies: residual immunogenicity resides in the CDR regions. MAbs. 2010;2(3):256-65.

9. Singh SK. Impact of product-related factors on immunogenicity of biotherapeutics. J Pharm Sci. 2011;100(2):354-87.

10. Chung CH, Mirakhur B, Chan E, Le QT, Berlin J, Morse M, et al. Cetuximab-induced anaphylaxis and $\mathrm{IgE}$ specific for galactosealpha-1,3-galactose. N Engl J Med. 2008;358(11):1109-17.

11. Steinke JW, Platts-Mills TA, Commins SP. The alpha-gal story: lessons learned from connecting the dots. J Allergy Clin Immunol. 2015;135(3):589-96.

12. Eli Lilly. ERBITUX ${ }^{\circledR}$ (cetuximab): prescribing information. May 2018. https://www.accessdata.fda.gov/drugsatfda_docs/label /2018/125084Orig1s268lbl.pdf. Accessed 4 Jun 2018.

13. Bosques CJ, Collins BE, Meador JW 3rd, Sarvaiya H, Murphy $\mathrm{JL}$, Dellorusso G, et al. Chinese hamster ovary cells can produce 
galactose-alpha-1,3-galactose antigens on proteins. Nat Biotechnol. 2010;28(11):1153-6.

14. Burton DR, Wilson IA. Immunology. Square-dancing antibodies. Science. 2007;317(5844):1507-8.

15. Salfeld JG. Isotype selection in antibody engineering. Nat Biotechnol. 2007;25:1369.

16. Jefferis R, Lefranc MP. Human immunoglobulin allotypes: possible implications for immunogenicity. MAbs. 2009;1(4):332-8.

17. Angal S, King DJ, Bodmer MW, Turner A, Lawson AD, Roberts $\mathrm{G}$, et al. A single amino acid substitution abolishes the heterogeneity of chimeric mouse/human (IgG4) antibody. Mol Immunol. 1993;30(1):105-8.

18. Aalberse RC, Schuurman J. IgG4 breaking the rules. Immunology. 2002;105(1):9-19.

19. Labrijn AF, Buijsse AO, van den Bremer ET, Verwilligen AY, Bleeker WK, Thorpe SJ, et al. Therapeutic IgG4 antibodies engage in Fab-arm exchange with endogenous human IgG4 in vivo. Nat Biotechnol. 2009;27(8):767-71.

20. van der Neut Kolfschoten M, Schuurman J, Losen M, Bleeker WK, Martinez-Martinez P, Vermeulen E, et al. Anti-inflammatory activity of human $\mathrm{IgG} 4$ antibodies by dynamic Fab arm exchange. Science. 2007;317(5844):1554-7.

21. Liu H, May K. Disulfide bond structures of IgG molecules: structural variations, chemical modifications and possible impacts to stability and biological function. MAbs. 2012;4(1):17-23.

22. Krishna M, Nadler SG. Immunogenicity to biotherapeutics-the role of anti-drug immune complexes. Front Immunol. 2016;7:21.

23. Ruhaak LR, Zauner G, Huhn C, Bruggink C, Deelder AM, Wuhrer $\mathrm{M}$. Glycan labeling strategies and their use in identification and quantification. Anal Bioanal Chem. 2010;397(8):3457-81.

24. Food and Drug Administration. Assay Development and Validation for Immunogenicity Testing of Therapeutic Protein Products. 2016. https://www.fda.gov/ucm/groups/fdagov-public/@fdago v-drugs-gen/documents/document/ucm192750.pdf. Accessed 30 Jul 2018.

25. European Medicines Agency. Guideline on Immunogenicity assessment of therapeutic proteins. 2017. http://www.ema.europ a.eu/docs/en_GB/document_library/Scientific_guideline/2017/06/ WC500228861.pdf. Accessed 21 Aug 2018.

26. Sampson HA, Munoz-Furlong A, Campbell RL, Adkinson NF Jr, Bock SA, Branum A, et al. Second symposium on the definition and management of anaphylaxis: summary report-Second National Institute of Allergy and Infectious Disease/Food Allergy and Anaphylaxis Network symposium. J Allergy Clin Immunol. 2006;117(2):391-7.

27. Qian J, Liu T, Yang L, Daus A, Crowley R, Zhou Q. Structural characterization of N-linked oligosaccharides on monoclonal antibody cetuximab by the combination of orthogonal matrix-assisted laser desorption/ionization hybrid quadrupole-quadrupole timeof-flight tandem mass spectrometry and sequential enzymatic digestion. Anal Biochem. 2007;364(1):8-18.

28. van Bueren JJL, Rispens T, Verploegen S, van der Palen-Merkus T, Stapel S, Workman LJ, et al. Anti-galactose- $\alpha$-1,3-galactose IgE from allergic patients does not bind $\alpha$-galactosylated glycans on intact therapeutic antibody Fc domains. Nat Biotechnol. 2011;29:574

29. Janin-Bussat MC, Tonini L, Huillet C, Colas O, Klinguer-Hamour $\mathrm{C}$, Corvaia N, et al. Cetuximab Fab and Fc N-glycan fast characterization using IdeS digestion and liquid chromatography coupled to electrospray ionization mass spectrometry. Methods Mol Biol. 2013;988:93-113

30. Gao B, Long C, Lee W, Zhang Z, Gao X, Landsittel D, et al. Anti-Neu5Gc and anti-non-Neu5Gc antibodies in healthy humans. PLoS One. 2017;12(7):e0180768.
31. Zhu A, Hurst R. Anti-N-glycolylneuraminic acid antibodies identified in healthy human serum. Xenotransplantation. 2002;9(6):376-81.

32. Kanda Y, Yamada T, Mori K, Okazaki A, Inoue M, Kitajima-Miyama $\mathrm{K}$, et al. Comparison of biological activity among nonfucosylated therapeutic IgG1 antibodies with three different $\mathrm{N}$-linked Fc oligosaccharides: the high-mannose, hybrid, and complex types. Glycobiology. 2007;17(1):104-18.

33. Wright A, Morrison SL. Effect of altered $\mathrm{CH} 2$-associated carbohydrate structure on the functional properties and in vivo fate of chimeric mouse-human immunoglobulin G1. J Exp Med. 1994;180(3):1087-96.

34. Wright A, Sato Y, Okada T, Chang K, Endo T, Morrison S. In vivo trafficking and catabolism of IgG1 antibodies with $\mathrm{Fc}$ associated carbohydrates of differing structure. Glycobiology. 2000;10(12):1347-55.

35. Yu M, Brown D, Reed C, Chung S, Lutman J, Stefanich E, et al. Production, characterization, and pharmacokinetic properties of antibodies with $\mathrm{N}$-linked mannose- 5 glycans. MAbs. 2012;4(4):475-87.

36. Zhou Q, Shankara S, Roy A, Qiu H, Estes S, McVie-Wylie A, et al. Development of a simple and rapid method for producing nonfucosylated oligomannose containing antibodies with increased effector function. Biotechnol Bioeng. 2008;99(3):652-65.

37. Luong M, Lam JS, Chen J, Levitz SM. Effects of fungal N- and O-linked mannosylation on the immunogenicity of model vaccines. Vaccine. 2007;25(22):4340-4.

38. Almond RJ, Flanagan BF, Kimber I, Dearman RJ. Influence of protein expression system on elicitation of $\operatorname{IgE}$ antibody responses: experience with lactoferrin. Toxicology. 2012;301(1-3):50-7.

39. Tatsumi Y, Sasahara Y, Kohyama N, Ayano S, Endo M, Yoshida $\mathrm{T}$, et al. Introducing site-specific glycosylation using protein engineering techniques reduces the immunogenicity of beta-lactoglobulin. Biosci Biotechnol Biochem. 2012;76(3):478-85.

40. Jefferis R. Posttranslational modifications and the immunogenicity of biotherapeutics. J Immunol Res. 2016;2016:5358272.

41. Schuurman J, Van Ree R, Perdok GJ, Van Doorn HR, Tan KY, Aalberse RC. Normal human immunoglobulin G4 is bispecific: it has two different antigen-combining sites. Immunology. 1999;97(4):693-8.

42. Koneczny I, Stevens JA, De Rosa A, Huda S, Huijbers MG, Saxena A, et al. IgG4 autoantibodies against muscle-specific kinase undergo Fab-arm exchange in myasthenia gravis patients. J Autoimmun. 2017;77:104-15.

43. Broug E, Bland-Ward PA, Powell J, Johnson KS. Fab-arm exchange. Nat Biotechnol. 2010;28(2):123-6.

44. Mullins RJ, Wainstein BK, Barnes EH, Liew WK, Campbell DE. Increases in anaphylaxis fatalities in Australia from 1997 to 2013. Clin Exp Allergy. 2016;46(8):1099-110.

45. Turner PJ, Gowland MH, Sharma V, Ierodiakonou D, Harper $\mathrm{N}$, Garcez $\mathrm{T}$, et al. Increase in anaphylaxis-related hospitalizations but no increase in fatalities: an analysis of United Kingdom national anaphylaxis data, 1992-2012. J Allergy Clin Immunol. 2015;135(4):956-63.

46. Hanania NA, Korenblat P, Chapman KR, Bateman ED, Kopecky $\mathrm{P}$, Paggiaro $\mathrm{P}$, et al. Efficacy and safety of lebrikizumab in patients with uncontrolled asthma (LAVOLTA I and LAVOLTA II): replicate, Phase 3, randomised, double-blind, placebo-controlled trials. Lancet Respir Med. 2016;4(10):781-96.

47. O'Neil BH, Allen R, Spigel DR, Stinchcombe TE, Moore DT, Berlin JD, et al. High incidence of cetuximab-related infusion reactions in Tennessee and North Carolina and the association with atopic history. J Clin Oncol. 2007;25(24):3644-8.

48. Cunningham D, Humblet Y, Siena S, Khayat D, Bleiberg H, Santoro A, et al. Cetuximab monotherapy and cetuximab plus 
irinotecan in irinotecan-refractory metastatic colorectal cancer. N Engl J Med. 2004;351(4):337-45.

49. Merck. ERBITUX ${ }^{\circledR}$ (cetuximab): summary of product characteristics. 19 Jul 2017. http://www.ema.europa.eu/ema/index .jsp?curl=pages $/$ medicines/human/medicines $/ 000558 /$ human _med_000769.jsp\&mid=WC0b01ac058001d124. Accessed 4 Jun 2018

50. Merck SDL. SIMPONI ${ }^{\circledR}$ (golimumab): summary of product characteristics. 19 Apr 2018. http://www.ema.europa.eu/ema/index .jsp?curl=pages/medicines/human/medicines/000992/human _med_001053.jsp\&mid=WC0b01ac058001d124. Accessed 25 May 2018.

51. Biogen Abbevie. ZINBRYTA ${ }^{\circledR}$ (daclizumab) prescribing information. 2017. https://www.zinbryta.com/content/dam/commercial/ multiple-sclerosis/zinbryta/pat/en_us/pdfs/zinbryta-prescribin g-information.pdf. Accessed 16 Jul 2018.

52. Janssen-Cilag Ltd. STELARA ${ }^{\circledR}$ (ustekinumab): summay of product characteristics. 22 Mar 2018. http://www.ema.europa.eu/ema/ index.jsp?curl=pages/medicines/human/medicines $/ 000958 /$ human _med_001065.jsp\&mid=WC0b01ac058001d124. Accessed 25 May 2018.
53. Novartis. ILARIS ${ }^{\circledR}$ (canakinumab): prescribing information. 2013. https://www.accessdata.fda.gov/drugsatfda_docs/label /2012/125319s0471bl.pdf. Accessed 20 Nov 2017.

54. Janssen Biotech, Inc. Golimumab prescribing information. 2018. https://www.janssenlabels.com/package-insert/product-monog raph/prescribing-information//SIMPONI-pi.pdf. Accessed 13 Jun 2018.

55. EMA. Ilaris-H-C-1109-II-0010: EPAR—assessment report-variation. 2013. http://www.ema.europa.eu/ema/index.jsp?curl=pages /medicines/human/medicines/001109/human_med_00082 6.jsp\&mid=WC0b01ac058001d124. Accessed 13 Jun 2018.

56. Zhang J, Li W, Roskos LK, Yang H. Immunogenicity assay cut point determination using nonparametric tolerance limit. J Immunol Methods. 2017;442:29-34.

57. European Medicines Agency. Guideline on similar biological medicinal products containing monoclonal antibodies-nonclinical and clinical issues. 30 May 2012. https://www.ema.europ a.eu/documents/scientific-guideline/guideline-similar-biological -medicinal-products-containing-monoclonal-antibodies-non-clini cal_en.pdf. Accessed 6 Dec 2018. 